\title{
MathRun: An Adaptive Mental Arithmetic Game Using A Quantitative Performance Model
}

\author{
Long Chen \\ Department of Creative Technology \\ Faculty of Science and Technology \\ Bournemouth University, Fern Barrow \\ Poole, Dorset, BH12 5BB \\ UK \\ chenl@bournemouth.ac.uk
}

\author{
Wen Tang \\ Department of Creative Technology \\ Faculty of Science and Technology \\ Bournemouth University, Fern Barrow \\ Poole, Dorset, BH12 5BB \\ UK \\ wtang@bournemouth.ac.uk
}

\begin{abstract}
Pedagogy and the way children learn are changing rapidly with the introduction of widely accessible computer technologies, from mobile apps to interactive educational games. Digital games have the capacity to embed many learning supports using the widely accredited VARK (visual, auditory, reading, and kinaesthetic) learning style. In this paper, we present a mathematics educational game MathRun for children age between 7-11 years old to practice mental arithmetic. We build the game as an interactive learning environment that fuses game mechanics with learning and uses the popular game genre "infinite runner" as the game mode. The game consists of an automatically generated infinite game map and mathematical questions also procedurally generated with varied levels of difficulty and complexity. A novel real-time performance evaluation method is developed for quantitative modeling the performance of the player. The model evaluates the performance in each primitive map block of the game map and level progression is automatically carried out based on the result of the evaluation. Therefore, the proposed game-based learning environment is adaptive to players with dynamic level progressions based on the combination of not only mathematics ability, but also gameplay skills of the player to facilitate learning processes through gameplay and appropriate adaptive progression of maths ability.
\end{abstract}

Educational Games; Incentive Structure in Games; Adaptive Level Progression; Game Design for Children;

\section{INTRODUCTION}

Games for education have been seen with great potential to motivate students. Recently the potential of games for learning at large scale has been recognised (Mayo, M. J., 2009). Maths games were designed to teach fundamental mathematics principals through games narratives, feedback and incentive structures (Martin et al, 2012). In this paper, we describe our mental arithmetic game called MathRun, which comprises arithmetical calculations currently with the target for Key Stage Curriculum (Gov.uk., 2014).

In MathRun student performs arithmetical calculations mentally without writing the figures down or using a calculator. Mental arithmetic is an important part of mathematics. It is also an essential part of coping with society's demands and managing everyday events (Department of Education UK, 2010). Mental arithmetical is an important element of key stage education, which is proven to be related to the math score of high school (Price et al , 2013).

Experiments have shown that the result of simple arithmetical problems such as single digit addition, subtraction and multiplication are fixed knowledge stored in a semantic memory network and usually can be directly retrieved without actual deliberate thinking and computation (Dehaene et al , 1997) (Zago el al , 2001). It has been proposed that these frequently-used simple questions, called arithmetic facts, can be learnt and stored as verbal associations mediated by language-based representations (Dehaene et al , 1992) (Klein, 2013).

Recent investigations also indicated that the process underlying the change from deliberate, conscious calculation to automatic and direct retrieval of answers from memory is actually caused by the long-term frequent practice. (Bourne et al , 1994) 


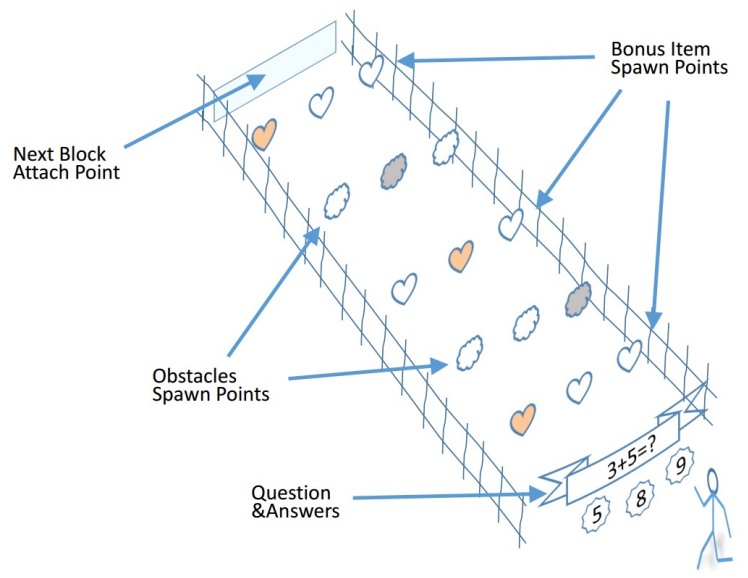

(a)

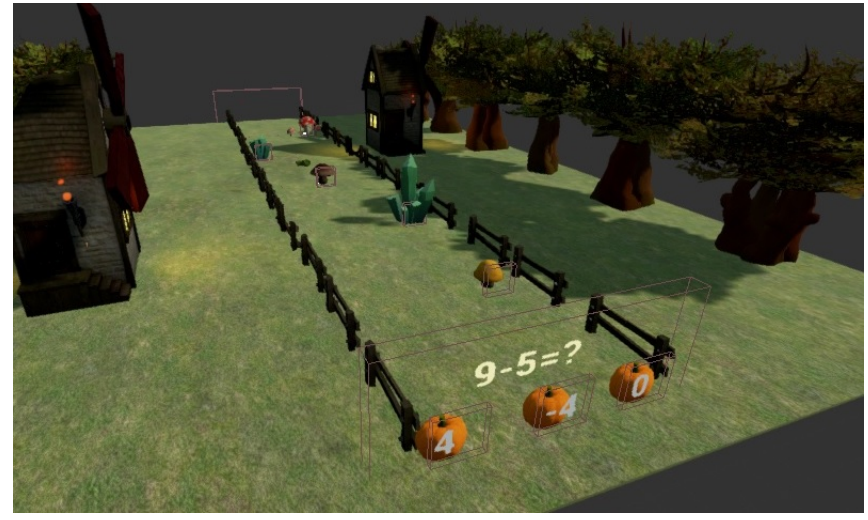

(b)

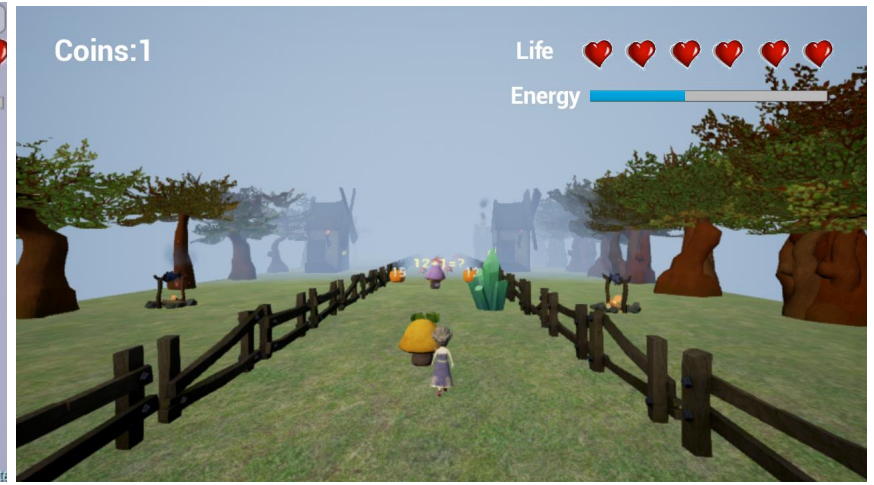

(d)

Figure 1: (a) The sketch of one map block, which consists of one question and three answers, three bonus items and two obstacles that randomly spawn at corresponding spawn points. (b) One map block in game editor; (c) The stitched map seen in a playing game with three primitive map blocks; (d) A screen capture in player's perspective.

(Pauli et al, 1994) The experiment results show that response time speeds up significantly across practice sessions, which follows the power law. More practice is completed. the quicker the problem gets processed.

There are a number of factors that children can derive and recall mental calculations, and children can learn a range of calculation strategies or methods that they can draw on. The educational game is a good way for children to learn while playing. The proposed game MathRun is designed to enable frequent practices through fun and motivation with a fast pace and infinite automatic game map methodology. To engage and motivate students to practice, an effective incentive structure in-game is vital to achieving this game-based learning objective.

Since every player has a different preference for the pace and style of game playing within a game (Charles et al, 2005), and the mental calculation ability between individuals varies even in the same year group (Dowker , 2005). Educational games usually have very narrow range of target students to avoid too simple for lower grade children or too hard for higher grade children, which greatly reduce the number of available game for the different level of children. It is vital to have an educational game that could cover a wider range of students without the need to select difficulty but to dynamically tailor the game to suit the ability of players. In addition, the game genre itself should be easy to control and popular among youths.

In this paper, we describe the development of a new 3D game for mental arithmetic practice based on the popular "infinite runner" game genre. We present a novel quantitative framework of measuring students in-game performance and incorporating learning behaviour while playing an educational game. Our quantitative framework is developed by introducing a performance model which dynamically guides the automatic generation of adaptive incentives for game-based learning of mathematical mental arithmetic. Our system generates rewards based on properties of mathematical model and student skills. The main contribution of this paper are: 
- A novel quantitative performance model that combines mathematical ability and gaming skills to generate motivation and engagement.

- Adaptive procedural level generation for mathematical mental arithmetic practice for a wide range of abilities.

- A new incentive system based on the quantitative performance model that can generate precise rewards and penalty points in-game play.

The MathRun game is designed based on the popular "infinite runner" game genre such as Temple Run (Imangi Studios, 2016) and Subway Surfers (Kiloo, 2016) which has seen more than 1 billion downloads (Tech Times, 2014). With simple game control and attractive gameplay mechanics, this type of games is very popular amongst young people aged between 6-25 years old. Therefore, not only employing such game genre in MathRun could draw interests to the target players of age between 711 years old, more importantly, the adaption of the fast pace of infinite runner is to meet the speed requirement for mental arithmetic gamebased learning.

We have developed MathRun as a third person 3D game with an essential gameplay that a the runner' character runs on an infinitely extensible road. The goal of the player is to survive as long as possible by collecting items to earn scores and avoid obstacles to reducing energy damage while answering mathematics questions correctly as shown in Figure 1 (a)). In contrast to Temple Run and Subway Surfers, the runner is not forced to run, but to control the movement of player character freely, so that the player is in control of the time to answer questions. Details of the game design methodology are described below, which include automatic map generation,core mathematics properties, life \& energy system, in-game performance evaluation, adaptive level switching, and the reward system.

\subsection{Map Generation}

We have designed a procedural game map generator to create infinity game map automatically. In addition, a method for generating random math questions and incentive elements in the map is also developed. A primitive map as a fundamental building block of the map is used to generate one question and three answers as shown in Figure 1 (a) and the corresponding scene in game editor Figure 1 (b). Figure 1 (c) illustrates an overview of the current map at a certain moment. The entire map is procedurally generated by stitching three primitive map blocks sequentially. The middle map block (with yellow border) is the map the player is currently

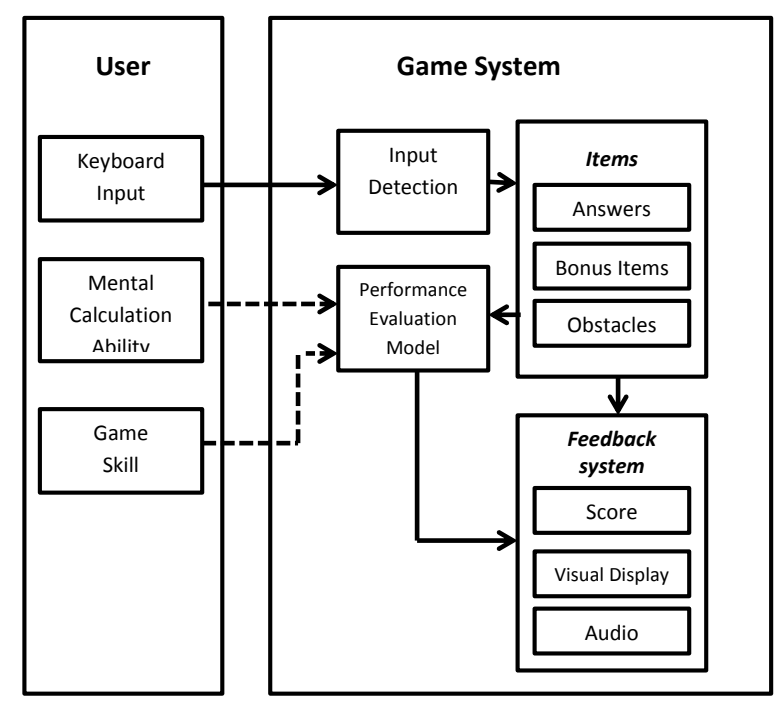

Figure 2: Overview of the user interaction and system.

standing on; the right map block will be deleted once the player has passed the map block, whilst the left map block is created. Figure 1 (d) shows a screen capture of the MathRun. The mythic fantasy world is created using special visual effects such as fog and animated assets.

With this procedural map generation process allows several advantages: the game can be played infinitely without manual design of a very large map; objects are created and destroyed dynamically to save computational resources; mathematical questions are created automatically and incentive items are created and controlled either randomly and precisely.

\subsection{Integration of Mathematical Questions}

To create a mathematical game as an infinite runner game, questions are designed as obstacles in the way of the player. Three answers are placed below each question so that the player needs to choose the correct answer to continue the game. Questions are derived from the National mathematics curriculum in England (Gov.uk., 2014), but the difficulty is slightly reduced with the expectation of mental calculation in this game and randomly generated based on the range designed for each current level; operators are also randomly generated with addition, subtraction, multiplication, and division; and correct or wrong answers are randomly selected from the positions of three pumpkins.

The current version of MathRun consists of 7 levels of difficulties with mathematical questions range from very simple $0 \sim 5$ addition/subtraction and $1 \sim 3$ multiplication/division to the difficult 3 numbers addition/subtraction ranges from $0 \sim 30$ and multiplication/division ranges from $2 \sim 5$. The 
Table 1: Level Design

\begin{tabular}{|c|c|c|}
\hline Level & Description & Example \\
\hline \multirow{2}{*}{1} & Addition or Subtraction $(0 \sim 5)$ & $2+4 ; 5-1 ;$ \\
\cline { 2 - 3 } & Multiplication or Division $(1 \sim 3)$ & $2 \times 3 ; 3 \div 1$ \\
\hline \multirow{2}{*}{2} & Addition or Subtraction $(0 \sim 9)$ & $5+7 ; 9-3$ \\
\cline { 2 - 3 } & Multiplication or Division $(1 \sim 5)$ & $3 \times 5 ; 4 \div 2$ \\
\hline \multirow{2}{*}{3} & Addition or Subtraction $(0 \sim 12)$ & $10+5 ; 12-7$ \\
\cline { 2 - 3 } & Multiplication or Division $(3 \sim 7)$ & $4 \times 7 ; 6 \div 3$ \\
\hline \multirow{2}{*}{4} & Addition or Subtraction $(0 \sim 20)$ & $11+15 ; 5-16$ \\
\cline { 2 - 3 } & Multiplication or Division $(5 \sim 9)$ & $5 \times 9 ; 9 \div 3$ \\
\hline \multirow{2}{*}{5} & Addition or Subtraction $(0 \sim 50)$ & $25+44 ; 37-28$ \\
\cline { 2 - 3 } & Multiplication or Division $(5 \sim 15)$ & $12 \times 11 ; 15 \div 3$ \\
\hline \multirow{2}{*}{6} & 3 Numbers Addition or Subtraction $(0 \sim 10)$ & $3+5+9 ; 7-5-3$ \\
\cline { 2 - 3 } & 3 Numbers Multiplication or Division $(1 \sim 3)$ & $2 \times 3 \times 3 ; 18 \div 3 \div 2$ \\
\hline \multirow{2}{*}{7} & 3 Numbers Addition or Subtraction $(0 \sim 30)$ & $10+15+21 ; 29-17-12$ \\
\cline { 2 - 3 } & 3 Numbers Multiplication or Division $(2 \sim 5)$ & $4 \times 5 \times 5 ; 90 \div 5 \div 3$ \\
\hline
\end{tabular}

detailed level design can be seen from Table 1 with example questions.

\subsection{Energy \& Life System}

In a normal infinite runner game, the player usually only controls the runner left, right and jump movements, and the forward movement of the player is controlled by the game. Instead of forcing the player to keep moving forward, in the MathRun, the player has the full freedom to control the pace of the movement so that there is sufficient time to complete the mathematical challenges. Therefore, the game flow in MathRun has been redesigned by adding an energy system to facilitate the gamebased education. The energy bar indicates the current energy decays along with time and If the energy is reduced to zero or the player selected a wrong answer, a life will be lost, and the energy will be refilled to full (see Figure 4(c) and (d)). Correct answers and picking up bonus items will boost the energy by $20 \%$ and $50 \%$ respectively (see the energy bar in Figure 4(a) and (b)) . If the energy exceeds $100 \%$, a life would be rewarded.

\section{QUANTITATIVE PERFORMANCE MODEL}

In order to apply adaptive difficulty in the MathRun game, for each map, we have designed a quantitative model to analyse the performance of player faced with the current difficulty. The model allows dynamic modifications of the difficulty of questions according to the player performance calculated by a performance score within each of the $i^{\text {th }}$ map block (see Figure 1 (a) for one map block):

$$
s_{i}=c_{i}+a \cdot b^{\left(t_{i}-T\right)}-\left(d_{i}-D\right)
$$

Equation 1 describes the performance model, where $c_{i}$ denotes the number of incentive items obtained in the $i^{\text {th }}$ map block, $t_{i}$ is the total time spent on the $i^{t h}$ map block with $\mathrm{T}$ as the minimal time required to complete the map block from the beginning to the end of the block. An estimated average value for $\mathrm{T}$ is about 6 seconds, therefore, $t_{i}-T$ represents the delay time of the player completing the block, which means the longer it takes to finish the map, the smaller score of the player to be awarded. We design $b$ as a constant value between 0 to 1 , thus mapping the effect of the time delay using the exponent of $b$ to $0 \sim 1$ and to avoid the performance score becoming negative. In our experiments, we set $b=$ 0.8 for a smoother mapping. in order to determine the effect of the time delay on the overall performance, a coefficient $a$ is used and based on our test, we set $a=5$. The last term in Equation 1 is the number of obstacles that the player runs into $d$, while $D$ stands for the number of obstacles in the map block, which is 2 in the current configuration. The final range of our performance score is between 0 to 15 (8 maximum number of coins in a map block, 5 for correct answers, and 3 for bonus items).

The performance score is awarded by taking into account of correct answers to the respond time to quantitatively assess the performance of the player in one map block, thus, offering guidance to adaptive level progressions and incentive elements delivery.

\section{ADAPTIVE LEVEL PROGRESSION FRAMEWORK}

The MathRun game is designed with UK 7-11 years old students as target players. The mental calculation ability between individuals varies even in the same year group (Dowker, 2005). Therefore, fixed level progression method will not be able to accommodate students with a variety of mental calculation abilities. More capable students should 


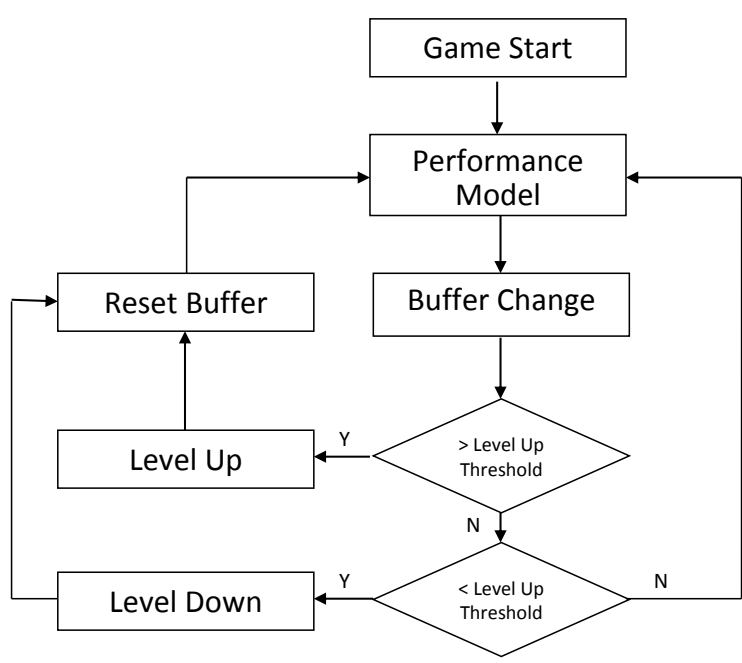

Figure 3: The adaptive level progression framework.

be charged and motivated while less able students should be encouraged to continuous their practice. The adaptive level progression is designed to make this game playable for a wider range of students ability.

Benefiting from our performance score model and automatic map generation system, the adaptive difficulty is very effectively implemented. The level difficulty will be increased as well as decreased automatically based on the player score analysis. As can be seen from the flow chart in Figure 3, the adaptive level progression makes the use of a level buffer for a smooth transition between levels. Table 2 summarises the level transaction process, in which the performance score of the current map block is mapped between -1 to +1 with the level buffer as 2 . When the level buffer reaches 1 or -1 , the game will be one level up or down and the level buffer is reset to 0 . The extreme performance score 15 or 0 will directly increase or reduce the game level. The middle range performance score will be given small value in the level buffer until it reaches the level switch threshold $(+1$ or -1$)$, minimizing the disturbance of performance score and to ensure a smooth adaptive level switching process.

\section{THE REWARD SYSTEM}

A multilevel reward system is designed in the MathRun to give reward, praise or penalty to the player based on the performance evaluation model.

On each map block level, different events can trigger reward or penalty points. Incentives take in different forms ranging from playing fancy graphics, sound to increase or decrease the energy and number of lives of the player. For example, obtaining a golden coin
Table 2: Performance Score to Level Buffer Mapping Table

\begin{tabular}{ccc}
\hline Score & & Effect to Buffer \\
\hline 0 & $\rightarrow$ & -1.0 \\
1 & $\rightarrow$ & -0.8 \\
2 & $\rightarrow$ & -0.6 \\
3 & $\rightarrow$ & -0.4 \\
4 & $\rightarrow$ & -0.2 \\
5 & $\rightarrow$ & -0.2 \\
6 & $\rightarrow$ & -0.1 \\
7 & $\rightarrow$ & -0.1 \\
8 & $\rightarrow$ & +0.1 \\
9 & $\rightarrow$ & +0.1 \\
10 & $\rightarrow$ & +0.2 \\
11 & $\rightarrow$ & +0.2 \\
12 & $\rightarrow$ & +0.4 \\
13 & $\rightarrow$ & +0.6 \\
14 & $\rightarrow$ & +0.8 \\
15 & $\rightarrow$ & +1.0 \\
\hline
\end{tabular}

and bonus items will trigger crispy coin sound, the wrong answer will cause an explosion of pumpkins with the painful sound of player, etc.

The performance of the player is also taken into account by comparing of the current map block with that performed on the previous map block to evaluate the performance in real-time taking into account of the mathematics ability and game skills. More importantly, based on long term gameplay performance, the system praises the player for persistence and an extra life is rewarded when the energy is full or when player collected a certain numbers of reward coins.

- Coin reward: One correct answer gains five coins. A wrong answer causes the reward coin and bonus items disappear (see Figure 4(d)).

- Lose of life: A wrong answer will make a life lost.

- Bonus items: Three bonus items are placed for the player to collect, if the question was answered correctly (see Figure 4(a) and (b) for picking a mushroom bonus item). Otherwise, the bonus items will disappear (see Figure 4(c) choose the wrong answer and (d) the pumpkin explodes along with the disappearance of bonus items).

- Energy Boost: On chosen of the correct answer and pick up bonus items, the energy will increase $20 \%$ and $50 \%$ respectively (see the energy bar in Figure 4(a) and (b)).

- Life Bonus (Energy): When player continuously picks the correct answers and bonus items with low respond time, the energy will exceed $100 \%$ 


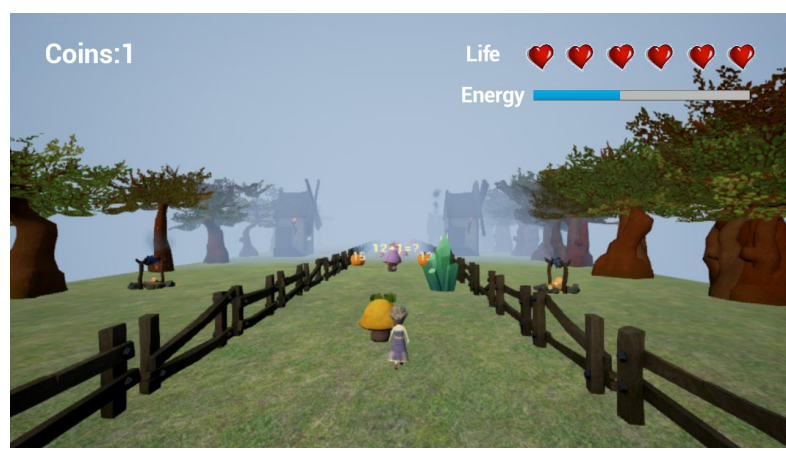

(a)

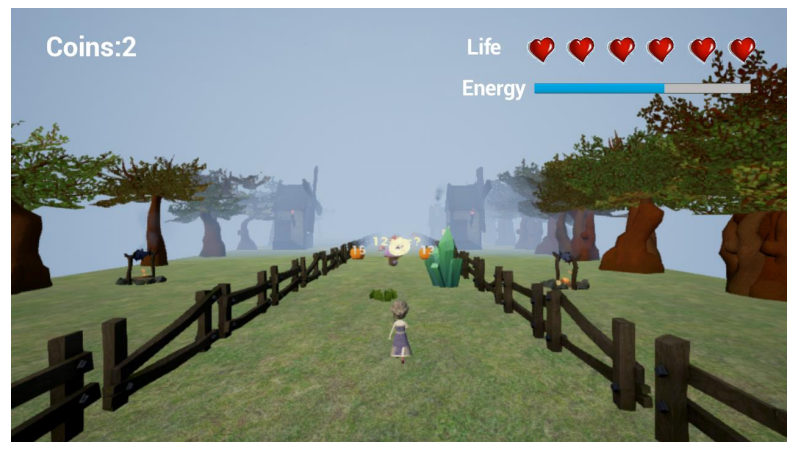

(b)

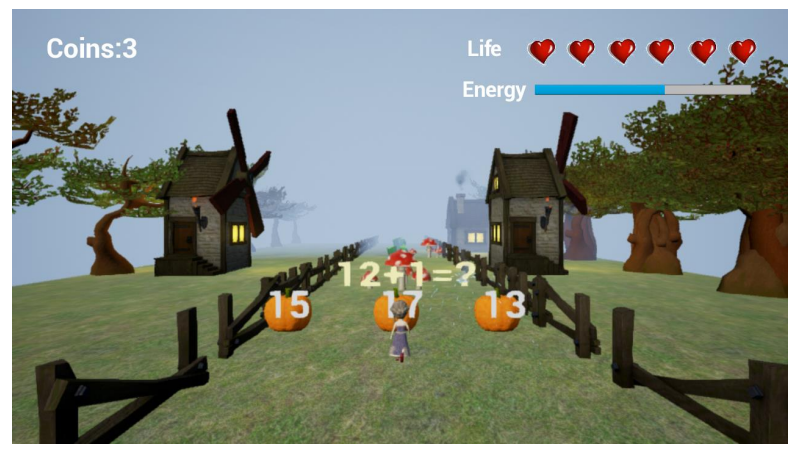

(c)

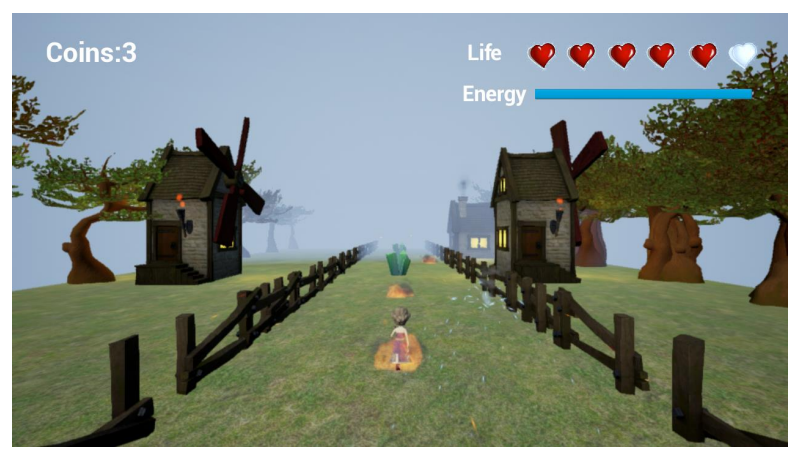

(d)

Figure 4: Game screen captures: (a) The player is going to pick a bonus item. (b) The player gets the bonus coin and gains $20 \%$ energy boots after collecting the bonus item. (c) The player is going to select a wrong answer. (d) The pumpkins exploded and the player lost one life after the wrong answer, along with the disappearance of the bonus item (mushroom). which can trigger the obtainment of an extra life.

- Life Bonus (Coin) : When player collects 100 coins, an extra life will be rewarded to prize the persistence.

- Level Switching Notice: Based on the level switching rule in Section 4, when the level progresses, the words "Congratulations, you step into Level XX !" will be displayed on the screen. Whereas when the level regresses, the words "Cheer up, you step back into Level XX !" will be shown on the screen.

These elaborate-designed incentive methods allow the user play the game in an encouraging environment, guiding the user to try their best to select the correct answer to practice the math mental calculation skills. While in the meantime, in the interval of two questions, collecting the random bonus items is an entertaining and encouraging process, which guarantee the user an interest in this game.

\section{RESULTS AND DISCUSSION}

We tested our adaptive level, the result can be found in Figure 5. The horizontal and vertical axes represent the round of questions (horizontal axes) and the performance score/level (left vertical axes) as well as the buffer value (right vertical axes). The blue curve denotes the performance score of each round that is in very high value in the beginning as the game level (represented by grey curve) starts with 2. The high score had a direct impact on the buffer value, which would cause the boost of the game level (1 to 22 round). The performance score dropped dramatically (after given several wrong answers) after the game level reaches 6, which result in the decrease of the level buffer. After the level buffer was lower than -1, the level degradation was triggered and the game level was dynamically adjusted to 5. After that, the performance score was raised again along with the rise of buffer and then game level. From this point, the game found the suitable level for this player and the game level came into a steady state of level 5 and level 6 .

The experiment results prove the effectiveness of our adaptive level progression model and its usefulness in the educational game. As we tested this adaptive level educational game by students with high mental calculation abilities, the game level was dynamically adjusted to be in a very high level. Therefore, for the students with medium or poor mental calculation abilities, the game level will be adaptively adjusted to medium or low levels. In this version of MathRun, many variables such as the 


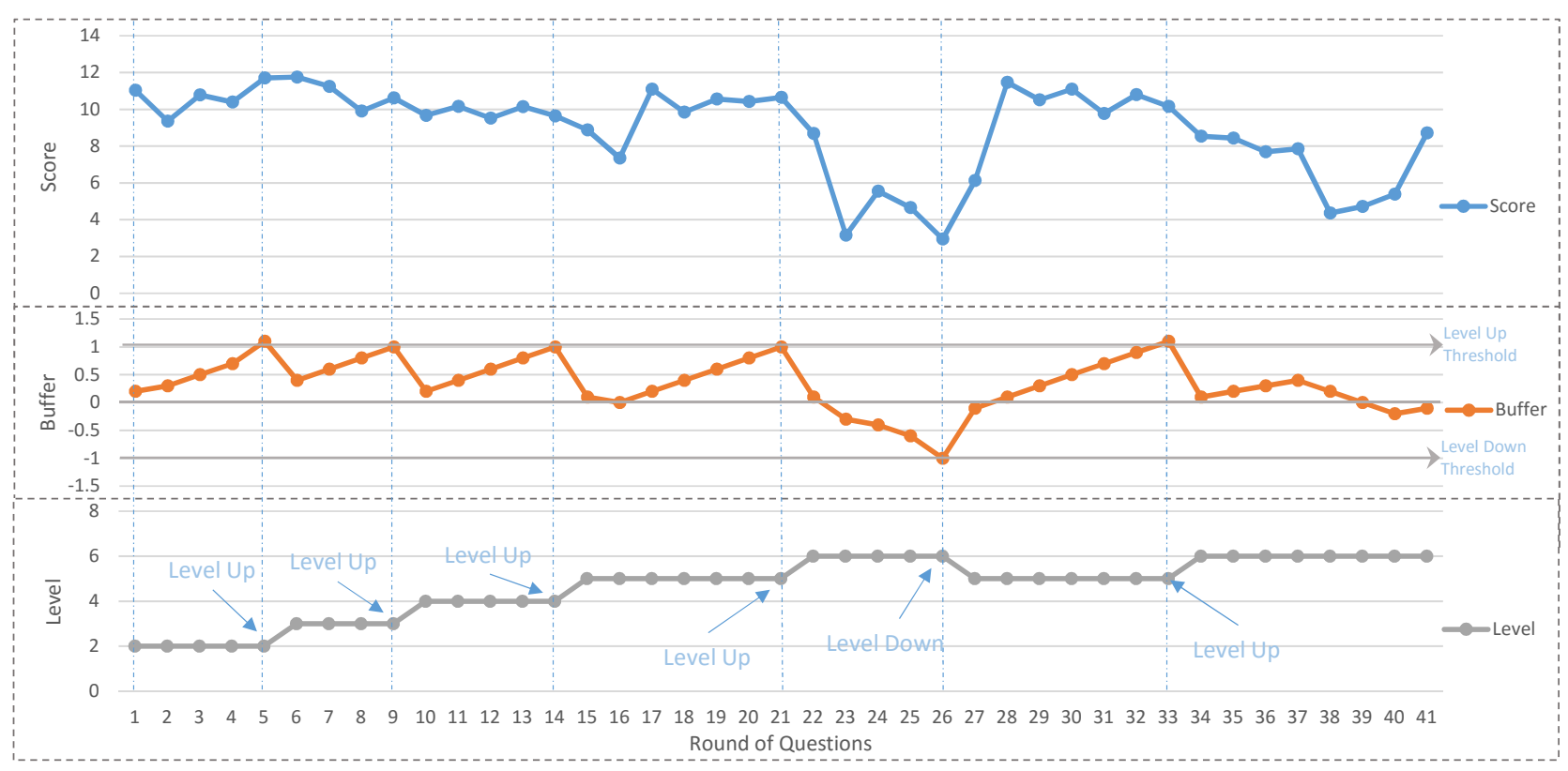

Figure 5: The experiment result shows the effectness of the adaptive level.

coefficients of coins, delay time, and obstacles as well as the mapping relationship of performance score and buffer change are selected based on our test or experience. Further work will involve the test of students with different mental calculation levels to evaluate our system and find a better mapping relationship between entertainment and learning.

\section{CONCLUSION}

A mental arithmetic game is presented based on the popular "infinite runner" game genre. With the quantitative performance model, real-time dynamic analysis of the player's performance of mental calculations can be carried out in-game along with performance evaluation of game skills for adaptive level progression. An automatic map generator is developed to create an efficient learning and gaming environment. The experiment results show that our proposed progression model can effectively switch levels to a suitable difficulty of mathematical questions that match the mental calculation ability of the player. Further study will involve a large scale user tests among a wider range of mental calculation abilities of students to evaluate the efficacy of the proposed game-based mental calculation practice.

\section{REFERENCES}

Bourne Jr, L. E., \& Rickard, T. C. (1991). Mental calculation: The development of a cognitive skill. In Inter-american Congress of Psychology, San Jose, Costa Rica.
Charles D, Kerr A, McNeill M, et al. (2005). Player-centred game design: Player modelling and adaptive digital games. Proceedings of the Digital Games Research Conference. Vol. 285.

Dehaene, S. (1997). The number sense. New York: Oxford University Press.

Dehaene, S. (1992). Varieties of numerical abilities. Cognition, 44(1-2), pp.1-42.

The National Strategies (2010) Primary: Teaching children to calculate mentally, Department for Education.

Dowker, A. (2005). Individual differences in arithmetic. Hove [U.K.]: Psychology Press.

Gov.uk. (2014). National curriculum in England: mathematics programmes of study - GOV.UK. [online] Available at: https://www.gov.uk/government/publications/nationalcurriculum-in-england-mathematics-programmesof-study/national-curriculum-in-englandmathematics-programmes-of-study [Accessed 31 May 2016].

Imangi Studios. (2016). Imangi Studios. [online] Available at: http://imangistudios.com/ [Accessed 29 May 2016].

Kiloo. (2016). Subway Surfers - Kiloo. [online] Available at: http://www.kiloo.com/games/subwaysurfers/ [Accessed 28 May 2016].

Klein, E., Moeller, K., Glauche, V., Weiller, C. and Willmes, K. (2013). Processing Pathways 
in Mental ArithmeticEvidence from Probabilistic

Fiber Tracking. PLoS ONE, 8(1), p.e55455.

Martin, T., Smith, C. P., Andersen, E., Liu, Y. E., \& Popovic, Z. (2012). Refraction time: Making split decisions in an online fraction game. In American Educational Research Association Annual Meeting, AERA.

Mayo, M. (2009). Video Games: A Route to LargeScale STEM Education?. Science, 323(5910), pp.79-82.

Pauli, P., Lutzenberger, W., Rau, H., Birbaumer, N., Rickard, T., Yaroush, R. and Bourne, L. (1994). Brain potentials during mental arithmetic: effects of extensive practice and problem difficulty. Cognitive Brain Research, 2(1), pp.21-29.

Price, G., Mazzocco, M. and Ansari, D. (2013). Why Mental Arithmetic Counts: Brain Activation during Single Digit Arithmetic Predicts High School Math Scores. Journal of Neuroscience, 33(1), pp.156163.

Tech Times. (2014). 'Temple Run' now in the billion download club. [online] Available at: http://www.techtimes.com/articles/7992/20140604/templerun-now-in-the-billion-download-club.htm [Accessed 29 May 2016].

Zago, L., Pesenti, M., Mellet, E., Crivello, F., Mazoyer, B. and Tzourio-Mazoyer, N. (2001). Neural Correlates of Simple and Complex Mental Calculation. Neurolmage, 13(2), pp.314-327. 